\title{
Voxelwise Detection of Cerebral Microbleed in CADASIL Patients by Genetic Algorithm and Back Propagation Neural Network
}

\author{
Yuanyuan Tao ${ }^{1}$ and Rayan S Cloutie ${ }^{2}$ \\ ${ }^{1}$ School of Computer Science and Technology, Nanjing Normal University, Nanjing, Jiangsu 210023, China \\ ${ }^{2}$ Department of Systems and Computer Engineering, Carleton University, Ottawa, ON K1S 5B6, Canada
}

\begin{abstract}
In this study, we proposed an application of voxelwise detection of cerebral microbleed in CADASIL patients by genetic algorithm (GA) and back propagation neural network (BPNN).We collected in total 20 subjects, and obtained 69,356 CMB voxels, and 124,063,981 non-CMB voxels. We employed BPNN as the classification tool. For BPNN has better prediction, we used GA to optimize BPNN. Finally, we used 10-fold cross validation to verify classifier performance. Our method obtained a sensitivity of $72.90 \pm 1.38 \%$, a specificity of $72.89 \pm 1.18 \%$, and an accuracy of $72.90 \pm 1.28 \%$.
\end{abstract}

Keywords - voxelwise detection; CADASIL; genetic algorithm; back propagation neural network

\section{INTRODUCTION}

In the field of medicine, the cerebral autosomal-dominant arteriopathy with subcortical infarcts and leukoencephalopathy (CADASIL) is a hereditary arteriopathy [1]. CADASIL is a hereditary cerebral vascular disease caused by a mutation in the Notch3 gene located on chromosome 19. The clinical manifestations of CADASIL are mainly migraine, subcortical ischemia, cognitive decline, dementia and mental symptoms, of which migraine is usually the earliest clinical manifestation.

The imaging manifestations of CADASIL patients mainly include white matter lesions, lacunar infarction, cerebral microbleed (CMB) and brain atrophy. In our study, we chose to detect CADASIL through imaging features of CMB.

Traditionally, doctors use images to judge manually. However, this is time consuming and error prone. Therefore, in order to assist doctors in manual CMB assessment, researchers in academia put forward advanced computer vision methods [24]. Chen, Yu (2015) [5] presented an automatic method via deep learning based 3D feature representation. Standvoss, Crijns (2018) [6] proposed a computer aided detection system based on 3D convolutional neural networks for detecting CMBs in 3D susceptibility weighted images. Dou, Chen (2015) [7] used a computer-aided system for automatic detection of CMBs from brain SWI images.

In this study, we used image processing and back propagation neural network (BPNN) techniques. We also used genetic algorithms (GA) [8-13]. GA is a kind of parallel stochastic search optimization method based on the simulation of natural genetic mechanism and biological evolution theory. The purpose of the GA is to optimize the initial weights and thresholds of the BP neural network and use the optimized network to make better predictions. In the experiment, we shall compare GA with simulated annealing algorithm (SAA) [14].

The overall structure of this paper is as follows: Section 2 offers the materials and methods. Section 3 provides results, and offers the discussions. Section 4 gives the conclusions.

\section{MATERIALS AND METHODS}

\section{A. Dataset}

In this study, we obtained 10 cerebral autosomal-dominant arteriopathy with subcortical infarcts and leukoencephalopathy (CADASIL) patients and 10 healthy controls (HCs). All the data are manually marked by professional doctors who have over 20 years of experience. Although manual detection may lead to errors, we have tried our best to minimize errors.

We collected in total 20 subjects, and obtained 69,356 CMB voxels, and 124,063,981 non-CMB voxels. Sliding-neighbor processing was used, i.e., picking up a small-size neighbor for each voxel. For class imbalance problem, we selected randomly 69,327 non-CMB voxels from those $124,063,981$ samples. Now we have a dataset as listed in Table 1.

TABLE I. OUR DATASET

\begin{tabular}{ll}
\hline Type & Number of samples \\
\hline CMB & 69,365 \\
Non-CMB & 69,327 \\
Total & 138,692 \\
\hline
\end{tabular}

B. Back Propagation Neural Network

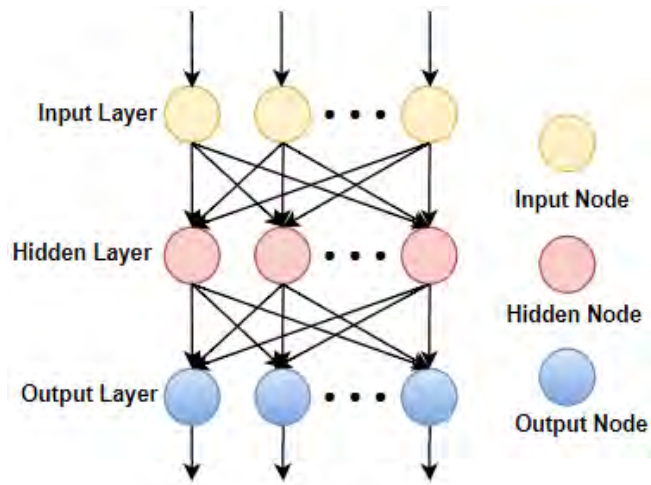

FIGURE I. STRUCTURE OF A BACK PROPAGATION NEURAL NETWORK 
Since the size of our dataset is quite small, there is no need to use any feature extraction $[15,16]$ or to do any feature reduction work $[17,18]$. The back propagation neural network (BPNN) is a multilayer feedforward neural network trained in accordance with the error reverse propagation algorithm, which is the most widely used neural network. The BPNN can continuously modify the connection weights among the artificial neurons that make up the forward multilayer network, so that the forward multilayer network can transform the information input into it into the desired output information [19]. The BPNN model structure is shown in Figure 1, which consists of an input layer, a hidden layer, and an output layer.

The process of BPNN is divided into two stages: (i) forward propagation: The input sample is processed layer by layer from the input layer through the hidden unit [20-22]. After passing through all hidden layers, it is passed to the output layer. (ii) backward propagation: The error signal is returned back in the direction of the original forward propagation path, and the weight coefficient of each neuron in each hidden layer is modified to minimize the error signal. In BPNN, our error formula is defined as follows:

$$
E_{\text {total }}=\sum \frac{1}{2}\left(Y_{\text {target }}-Y_{\text {output }}\right)^{2}
$$

where $E_{\text {total }}$ represents total errors, $Y_{\text {target }}$ represents the target results and $Y_{\text {output }}$ represents output results. Our goal is to minimize the total error. Back propagation updates parameters $\mathrm{w}$ and $\mathrm{b}$ by gradient descent method. The update formulas for parameters $\mathrm{w}$ and $\mathrm{b}$ are as follows:

$$
\begin{gathered}
\mathrm{w}=\mathrm{w}-\delta \frac{\partial E_{\text {total }}}{\partial w} \\
\mathrm{~b}=\mathrm{b}-\delta \frac{\partial E_{\text {total }}}{b}
\end{gathered}
$$

where $\delta$ represents learning rate.

\section{Genetic Algorithm}

Genetic algorithm (GA) is a randomized search method that evolved from the evolutionary rules of the biological world [23]. Based on the biological evolutionary principle of "survival of the fittest and survival of the fittest" in nature, a coded tandem population of optimized parameters is formed, and individuals are screened according to the selected fitness function by selection, crossover, and mutation in the inheritance so that the fitness value is good [24, 25]. Individuals are retained, individuals with poor fitness are eliminated, and new groups inherit both the previous generation's information and the previous generation. Repeatedly cycle until conditions are met.

GA is also a search heuristic algorithm for solving optimization in the field of artificial intelligence, and is a kind of evolutionary algorithm. This heuristic is often used to generate useful solutions to optimize and search for problems. Evolutionary algorithms were originally developed by drawing lessons from some of the phenomena of evolutionary biology, including genetics, mutations, natural selection, and crossbreeding. There are some other bioinspired algorithm, e.g., particle swarm optimization [26-29], biogeography-based optimization [30-33],

In our study, we use GA to optimize BPNN, including neural network structure determination, GA optimization, and BPNN prediction. GA is used to optimize the initial weights and thresholds of BP neural network [34], so that the optimized BP neural network can better predict the function output. The purpose of GA to optimize BPNN is to obtain better network initial weights and thresholds through GA. The basic idea is to use the initial weights and thresholds of individuals to represent the network and the prediction errors of BPNN initialized by individual values as the individual's fitness value, through the selection, crossover, mutation operation to find the best individual, that is, the optimal BPNN initial weight. In this study, our fitness function is as follows:

$$
\mathrm{F}=\mathrm{k}\left(\sum_{i=1}^{n}\left|y_{i}-o_{i}\right|\right)
$$

where $\mathrm{n}$ is the output node number of the network, $y_{i}$ is the expected output of the ith node of the BPNN and $o_{i}$ is the actual output of the ith node and $\mathrm{k}$ is the coefficient. The flow chart of BPNN algorithm optimized by GA is shown in Figure 2.

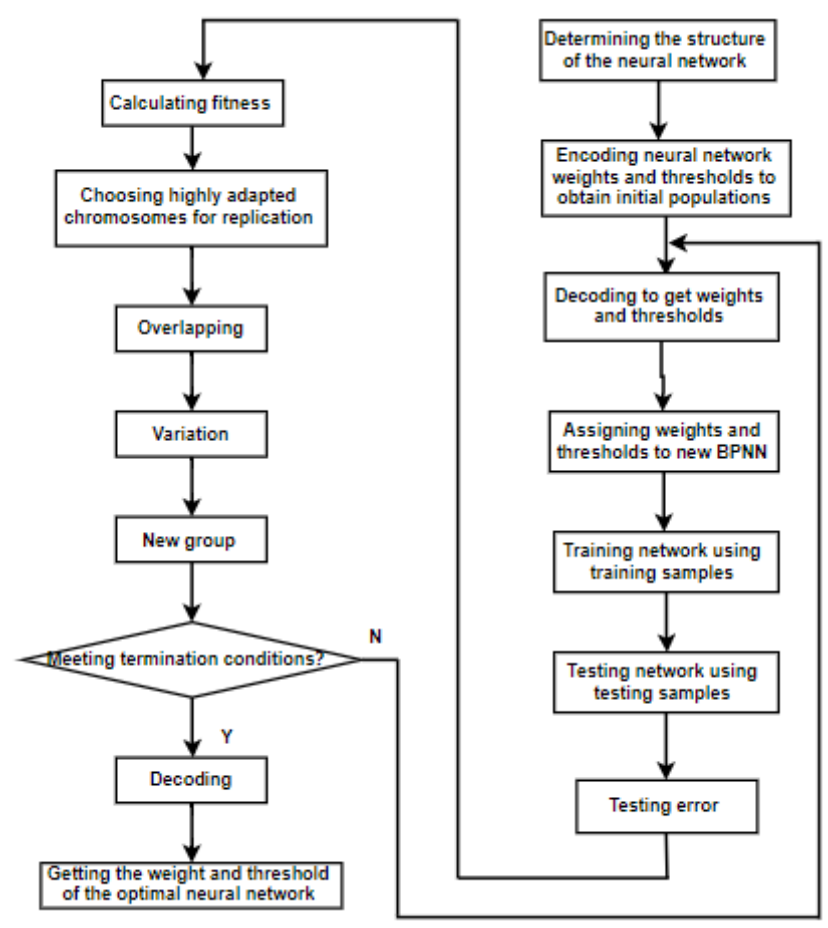

FIGURE II. THE FLOW CHART OF BPNN ALGORITHM OPTIMIZED BY GA

\section{EXPERIMENTS AND RESULTS}

We designed a 10-fold cross validation for our experiment. An illustration is shown in Figure 3. The orange color shows the training data, and other different colors represent one type of fold (in total 10 folds). This 10 -fold cross validation will repeat ten times, and we report the final mean and standard deviation of sensitivity, specificity, and accuracy. 


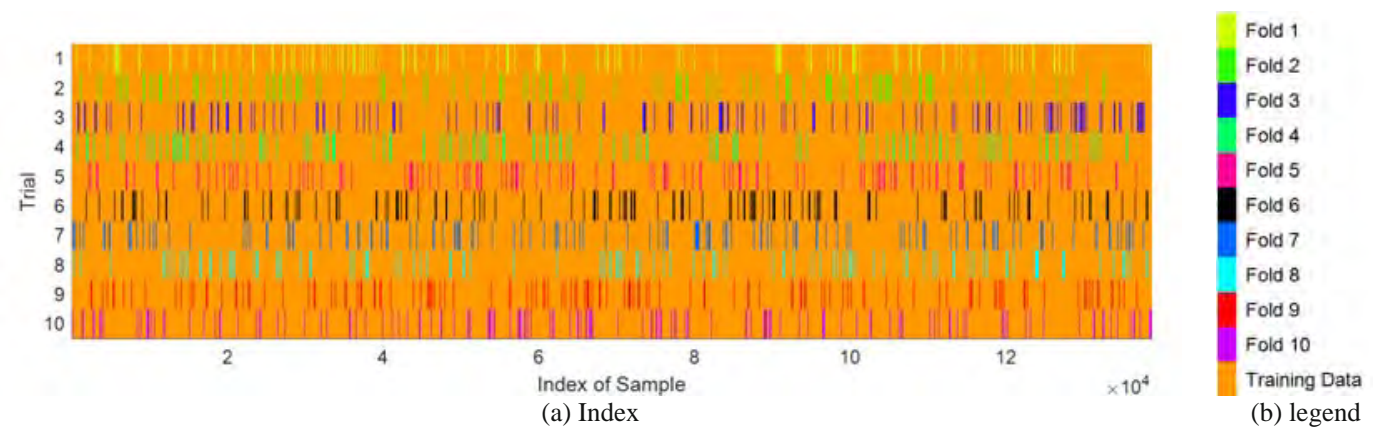

FIGURE III. ILLUSTRATION OF 10-FOLD CROSS VALIDATION

The performance of our GA-BPNN is shown in Table 2. Our method obtained a sensitivity of $72.90 \pm 1.38 \%$, a specificity of $72.89 \pm 1.18 \%$, and an accuracy of $72.90 \pm 1.28 \%$. Figure 4 shows the performance in bars. The red bar shows the average result, and the rest blue bars show the result of each run.

TABLE II. PERFORMANCE OF OUR GA-BPNN APPROACH

\begin{tabular}{|c|c|c|c|c|c|c|c|c|c|c|c|}
\hline Sen & F1 & $\mathrm{F} 2$ & F3 & $\mathrm{F} 4$ & F5 & F6 & F7 & F8 & F9 & F10 & Total \\
\hline 1 & & & & & & & & & & & 3.1 \\
\hline & & & & & & & & & & & \\
\hline 3 & & & & & & & & & & & 73.42 \\
\hline 4 & 74. & & & & & & & & & & 72.64 \\
\hline 5 & 72. & & & & & & & & & & 72.89 \\
\hline 6 & 72.8 & & & & & & & & & & 72.01 \\
\hline 7 & 73.3 & & 77. & 72. & & 67 & & 71. & & & 72.51 \\
\hline 8 & 77.6 & & & & & 74 & & 75 & & 10.00 & 75.44 \\
\hline 9 & 71. & & 13. & 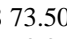 & & 11 & & 73 & & 14. & 74.32 \\
\hline R10 & 74. & 72. & 68.6 & 73. & 71. & 74 & & 71 & & 71.6 & 72.36 \\
\hline Spc & F1 & $\mathrm{F} 2$ & F3 & $\mathrm{F} 4$ & F5 & F6 & F7 & F8 & F9 & F10 & Total \\
\hline $\mathrm{R} 1$ & 73.3 & 72.62 & 71.37 & 76.2 & 68. & 74.7 & 72 . & 73.6 & +73 & 73.70 & 73.03 \\
\hline 2 & 70. & & & & & & & & & & 1.05 \\
\hline 3 & 73. & & & & & & & & & $/ 2$. & 73.33 \\
\hline 4 & 70.8 & & 70. & & & 13 & & 71. & & 71.8 & 72.55 \\
\hline 5 & 74. & & & & & & & /1 & & 40 & 72.73 \\
\hline 6 & $71.3^{3}$ & & & & & & & & & & 71.97 \\
\hline 7 & 72. & & & & & & & & & 13. & 72.29 \\
\hline & 72. & & & & & & & & & 77.6 & 75.28 \\
\hline & 74. & & 73. & & & & & & & & 74.21 \\
\hline R10 & 72.5 & 70. & 70.37 & 72.48 & 32.4 & 573.0 & 73.36 & 70.16 & 74.90 & 74.08 & 72.47 \\
\hline Acc & F1 & F2 & F3 & $\mathrm{F} 4$ & F5 & F6 & F7 & F8 & F9 & F10 & Total \\
\hline $\mathrm{R} 1$ & 73 & 73.7 & 71.6 & $74.3^{3}$ & 71. & 73. & 7 & 74 & 73 & 73.6 & 73.11 \\
\hline 2 & 71. & & & & & & & & & & 0.65 \\
\hline 3 & 72.7 & & & 72. & & & & & & 72.2 & 73.38 \\
\hline & 72. & $72 t$ & 72. & 71.0 & 72. & 73 & & 72.6 & & 71.8 & 72.60 \\
\hline & 73.7 & & 71. & 72. & 73. & 72 & & 71 & & 72. & 72.81 \\
\hline R6 & 72. & & 723 & 71.5 & & 72 & & 70. & & 68.9 & 71.99 \\
\hline R7 & 72.9 & & & 72.60 & 71. & 69. & & 70.36 & & 73.57 & 72.40 \\
\hline & 75.2 & & 74.77 & 75. & 71. & 74 & & 74 & & 77.2 & 75.36 \\
\hline & 72.8 & & 73. & 74. & 75. & 33. & & 73. & & 74.5 & 74.27 \\
\hline & & & & & & & & & & 72.87 & 72.41 \\
\hline
\end{tabular}
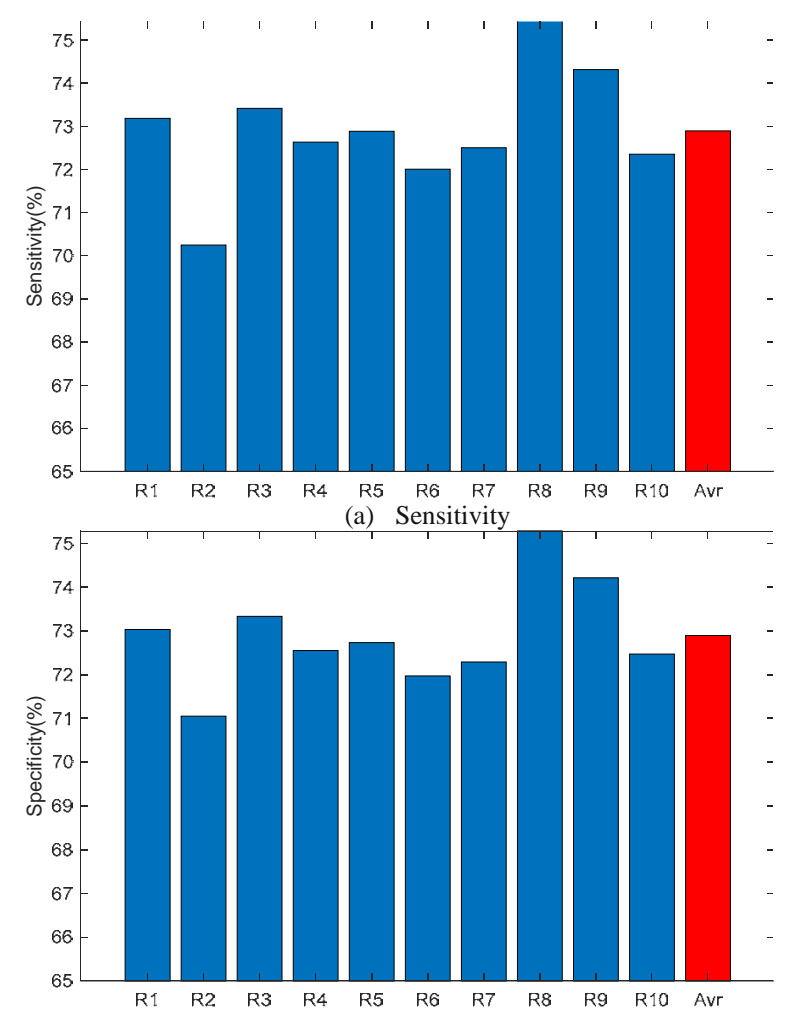

(b) Specificity

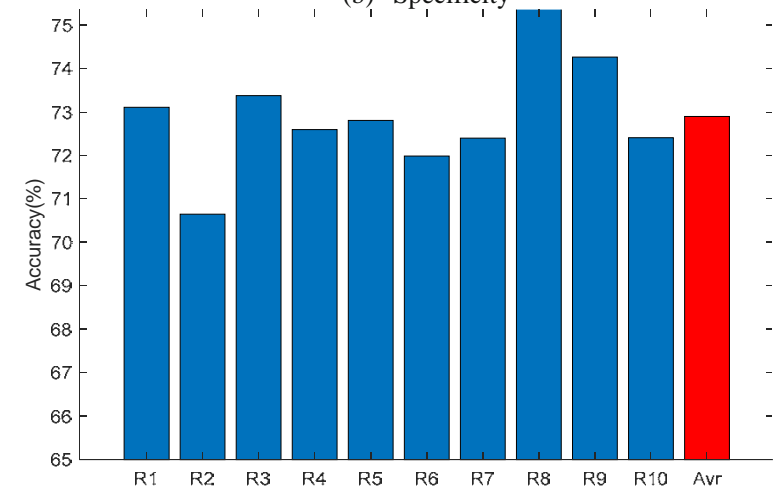

(c) Accuracy

FIGURE IV. PERFORMANCE OF OUR METHOD

Finally, we compared our genetic algorithm with simulated annealing algorithm (SAA) [14]. The results are shown below 
in Table 3. We can observe that our GA-BPNN has obtained better performance than SAA-BPNN. Our GA-BPNN may apply to other diseases, such as multiple sclerosis [35], alcoholism [36],

\section{TABLE III. ALGORITHM COMPARISON}

\begin{tabular}{|l|l|l|l|}
\hline Approach & Sensitivity & Specificity & Accuracy \\
\hline SAA-BPNN [14] & $68.45 \pm 1.26$ & $68.47 \pm 1.39$ & $68.46 \pm 1.22$ \\
\hline GA-BPNN (Our) & $72.90 \pm 1.38$ & $72.89 \pm 1.18$ & $72.90 \pm 1.28$ \\
\hline
\end{tabular}

\section{CONCLUSION}

In this paper, we developed a new voxelwise CMB detection system for CADASIL patients. The results showed that the proposed method gave better performance than BPNN optimized by SAA.

In the future, we may focus on following regards: (i)we shall either collect more brain images or use data augmentation techniques. (ii) Some other advanced classifiers may be used, such as convolutional neural network (CNN). (iii) Our method may apply to $x$-ray image segmentation [37]. All of the above are the directions of our future research.

\section{REFERENCES}

[1] Schubert, V., et al., A novel frameshift variant in the CADASIL gene NOTCH3: pathogenic or not? Journal of Neurology, 2018. 265(6): p. 1338-1342

[2] Lu, S. Detection of cerebral microbleeding based on deep convolutional neural network. in 14th International Computer Conference on Wavelet Active Media Technology and Information Processing (ICCWAMTIP). 2017. Chengdu, China: IEEE. p. 93-96

[3] Hou, X.-X., Seven-layer deep neural network based on sparse autoencoder for voxelwise detection of cerebral microbleed. Multimedia Tools and Applications, 2018. 77(9): p. 10521-10538

[4] Hou, X.-X., Voxelwise detection of cerebral microbleed in CADASIL patients by leaky rectified linear unit and early stopping. Multimedia Tools and Applications, 2017, doi: 10.1007/s11042-017-4383-9.

[5] Chen, H., et al. Automatic detection of cerebral microbleeds via deep learning based $3 D$ feature representation. in 12th International Symposium on Biomedical Imaging (ISBI). 2015. New York, NY, USA: IEEE. p. 764-767

[6] Standvoss, K., et al., Cerebral Microbleed Detection in Traumatic Brain Injury Patients using 3D Convolutional Neural Networks. Conference on Medical Imaging - Computer-Aided Diagnosis, 2018. 10575: Article ID. UNSP 105751D

[7] Dou, Q., et al. Automatic Cerebral Microbleeds Detection from MR Images via Independent Subspace Analysis Based Hierarchical Features. in 37th Annual International Conference of the IEEE Engineering in Medicine and Biology Society (EMBC). 2015. Milan, ITALY: IEEE. p. 7933-7936

[8] Lu, S., A note on the weight of inverse complexity in improved hybrid genetic algorithm. Journal of Medical Systems, 2016. 40(6): Article ID. 150

[9] Wu, L. Recursive structure element decomposition using migration fitness scaling genetic algorithm. in 2nd International Conference on Swarm Intelligence (ICSI). 2011. Chongqing, China: Springer Verlag. p. 514-521

[10] Li, J., Texture Analysis Method Based on Fractional Fourier Entropy and Fitness-scaling Adaptive Genetic Algorithm for Detecting Left-sided and Right-sided Sensorineural Hearing Loss. Fundamenta Informaticae, 2017. 151(1-4): p. 505-521

[11] Huo, Y. Predict Two-Dimensional Protein Folding Based on Hydrophobic-Polar Lattice Model and Chaotic Clonal Genetic Algorithm. in International Conference on Intelligent Data Engineering and Automated Learning (IDEAL). 2016. Yangzhou, China: Springer International Publishing. p. 10-17
[12] Wei, L., et al., Fitness-scaling adaptive genetic algorithm with local search for solving the Multiple Depot Vehicle Routing Problem. Simulation, 2016. 92(7): p. 601-616

[13] Wu, L.N. Bankruptcy Prediction by Genetic Ant Colony Algorithm. in International Conference on Computer-aided Material and Engineering. 2011. Hangzhou, PEOPLES R CHINA: Trans Tech Publications Ltd. p. 459-463

[14] Bagherlou, H., et al., A routing protocol for vehicular ad hoc networks using simulated annealing algorithm and neural networks. Journal of Supercomputing, 2018. 74(6): p. 2528-2552

[15] Yang, J.F., Comprehensive Survey on Fractional Fourier Transform. Fundamenta Informaticae, 2017. 151(1-4): p. 1-48

[16] Han, L., Identification of Alcoholism based on wavelet Renyi entropy and three-segment encoded Jaya algorithm. Complexity, 2018. 2018: Article ID. 3198184

[17] Yan, J., A note on Das's PCA in online phases. Progress in Electromagnetics Research Letters, 2015. 51: p. 117-118

[18] Wu, L., An MR brain images classifier via principal component analysis and kernel support vector machine. Progress In Electromagnetics Research, 2012. 130: p. 369-388

[19] Chiba, Z., et al., A novel architecture combined with optimal parameters for back propagation neural networks applied to anomaly network intrusion detection. Computers \& Security, 2018. 75: p. 36-58

[20] Zhan, T., Pathological brain detection by artificial intelligence in magnetic resonance imaging scanning. Progress in Electromagnetics Research, 2016. 156: p. 105-133

[21] Li, Y., Detection of Dendritic Spines using Wavelet Packet Entropy and Fuzzy Support Vector Machine. CNS \& Neurological Disorders - Drug Targets, 2017. 16(2): p. 116-121

[22] Li, Y.-J., Single slice based detection for Alzheimer's disease via wavelet entropy and multilayer perceptron trained by biogeography-based optimization. Multimedia Tools and Applications, 2018. 77(9): p. 1039310417

[23] Alisi, I.O., et al., Quantitative Structure Activity Relationship Analysis of Coumarins as Free Radical Scavengers by Genetic Function Algorithm. Physical Chemistry Research, 2018. 6(1): p. 208-222

[24] Ji, G., Genetic Pattern Search and Its Application to Brain Image Classification. Mathematical Problems in Engineering, 2013: Article ID. 580876

[25] Ji, G.L., A Rule-Based Model for Bankruptcy Prediction Based on an Improved Genetic Ant Colony Algorithm. Mathematical Problems in Engineering, 2013: Article ID. 753251

[26] Jun, Y., et al., Find multi-objective paths in stochastic networks via chaotic immune PSO. Expert Systems with Applications, 2010. 37(3): p. 1911-1919

[27] Wu, L., Crop Classification by forward neural network with adaptive chaotic particle swarm optimization. Sensors, 2011. 11(5): p. 4721-4743

[28] Wu, L., UCAV path planning by fitness-scaling adaptive chaotic particle swarm optimization. Mathematical Problems in Engineering, 2013: Article ID. 705238

[29] Ji, G., An MR brain images classifier system via particle swarm optimization and kernel support vector machine. The Scientific World Journal, 2013: Article ID. 130134

[30] Phillips, P., Pathological brain detection in magnetic resonance imaging scanning by wavelet entropy and hybridization of biogeography-based optimization and particle swarm optimization. Progress In Electromagnetics Research, 2015. 152: p. 41-58

[31] Wu, J., Fruit classification by biogeography-based optimization and feedforward neural network. Expert Systems, 2016. 33(3): p. 239-253

[32] Wu, X., Smart detection on abnormal breasts in digital mammography based on contrast-limited adaptive histogram equalization and chaotic adaptive real-coded biogeography-based optimization. Simulation, 2016. 92(9): p. 873-885

[33] Li, P., et al., Pathological Brain Detection via Wavelet Packet Tsallis Entropy and Real-Coded Biogeography-based Optimization. Fundamenta Informaticae, 2017. 151(1-4): p. 275-291

[34] Murthy, Y.V.S., et al., Classification of vocal and non-vocal segments in 
audio clips using genetic algorithm based feature selection (GAFS). Expert Systems with Applications, 2018. 106: p. 77-91

[35] Dong, Z., Synthetic Minority Oversampling Technique and Fractal Dimension for Identifying Multiple Sclerosis. Fractals, 2017. 25(4): Article ID. 1740010

[36] Hou, X.-X., Alcoholism detection by medical robots based on Hu moment invariants and predator-prey adaptive-inertia chaotic particle swarm optimization. Computers and Electrical Engineering, 2017. 63: p. 126-138

[37] Lu, S.Y., A note on the marker-based watershed method for X-ray image segmentation. Computer Methods and Programs in Biomedicine, 2017. 141: p. $1-2$ 